\title{
Ordinal proximity measures in the context of unbalanced qualitative scales and some applications to consensus and clustering
}

\author{
José Luis García-Lapresta \\ PRESAD Research Group, IMUVA, Dept. de Economía Aplicada, Universidad de \\ Valladolid, Spain \\ David Pérez-Román \\ PRESAD Research Group, Dept. de Organización de Empresas y Comercialización e \\ Investigación de Mercados, Universidad de Valladolid, Spain
}

\begin{abstract}
In this paper, we introduce ordinal proximity measures in the setting of unbalanced qualitative scales by comparing the proximities between linguistic terms without numbers, in a purely ordinal approach. With this new tool, we propose how to measure the consensus in a set of agents when they assess a set of alternatives through an unbalanced qualitative scale. We also introduce an agglomerative hierarchical clustering procedure based on these consensus measures.

Keywords: decision making; qualitative scales; proximity; difference measurement; consensus; clustering.
\end{abstract}

\section{Introduction}

In different decision-making problems, agents have to show their opinions on a set of alternatives and then an aggregation procedure is used for generating a collective outcome: a winning alternative, several winning alternatives, a ranking on the set of alternatives, etc.

The agents opinions can be provided in very different ways: the favorite alternative, a subset of acceptable alternatives, a ranking on the set of alternatives, an assessment for each alternative, etc.

When agents assess independently each alternative, the corresponding assessments can be of different nature depending on the context: numerical values, intervals of real numbers, fuzzy numbers, linguistic terms, etc.

Email addresses: lapresta@eco.uva.es (José Luis García-Lapresta), david@emp.uva.es (David Pérez-Román)

Preprint submitted to Applied Soft Computing

January 23, 2015 
Qualitative scales are formed by linguistic terms. Usually, these scales are balanced and uniform: there are the same number of positive and negative terms, and adjacent terms are equidistant (for instance, 'very bad', 'bad', 'acceptable', 'good' and 'very good'). However, sometimes the qualitative scales are unbalanced: there are different number of positive terms compared to negative ones ${ }^{1}$, and it is not clear how to measure the nearness between the linguistic terms of an unbalanced qualitative scale ${ }^{2}$.

In this paper, we do not assign numerical distances between linguistic terms, but we propose to make pairwise comparisons of psychological proximities between them. This approach has some similarities with difference measurement within the classical measurement theory (see Krantz et al. [32, chapter 4] and Roberts [40, section 3.3]), and also with non-metric multidimensional scaling, where only the ranks of the psychological distances or proximities are known (see Bennett and Hays [5], Shepard [41], Coombs [12], Kruskal and Wish [33], Cox and Cox [13] and Borg and Groenen [7, chapter 9], among others). We have also to mention Bossert et al. [9] that consider ordinal measures of distances in the analysis of diversity.

In order to explain how the mentioned comparisons can be made, we consider, as an example, that some journals use the linguistic terms 'reject', 'major revision', 'minor revision' and 'accept' in the evaluation of papers. It has no sense to assign numerical values neither to these terms nor to distances between terms. However, an author may feel that the psychological proximity between 'minor revision' and 'accept' is bigger than the psychological proximity between 'minor revision' and 'major revision'. Obviously, this author could compare psychological proximities between the rest of pairs of linguistic terms. Initially, this task may seem hard, because there are $16^{2}=256$ possible pairwise comparisons. Fortunately, it is not necessary to compare all the pairs ${ }^{3}$ : the psychological proximity between two terms does not depend on the order these terms are presented; the psychological proximity between a term and itself is always the same and it is bigger than the psychological proximity between two different terms; etc.

Taking into account the previous ideas, we propose the notion of ordinal proximity measure as a mapping that assigns an element of a chain to each pair of psychological proximities between linguistic terms, satisfying four independent properties: all the elements in the chain correspond to some psychological proximity, i.e., no element in the chain is superfluous; psychological proximities are symmetric, i.e., the order of the pairs is irrelevant in the comparison; the maximum psychological proximity is reached when comparing a linguistic term with itself; and given three different linguistic terms, the degree of proximity

\footnotetext{
${ }^{1}$ For instance, Herrera et al. [24] consider the following nine linguistic terms: 'none', 'low', 'medium', 'almost high', 'high', 'quite high', 'very high' and 'total'.

${ }^{2}$ Nevertheless, within a fuzzy approach, some cardinal proposals on unbalanced qualitative scales can be found in Herrera et al. [24] and Cabrerizo et al. [11], among others.

${ }^{3}$ In Remark 1 we show that, with four linguistic terms, only between three and six comparisons are needed.
} 
between the lowest and the highest terms should be smaller than the degrees of proximity between the lowest and the intermediate terms and between the intermediate and the highest terms.

Once the ordinal proximity measuring model has been introduced, we propose consensus measures and agglomerative hierarchical clustering procedures when a group of agents evaluate the alternatives through a qualitative scale, taking into account the ordinal proximities between individual assessments.

Given a subset of agents and a subset of alternatives, we define the degree of consensus as the upper median of the proximities between all the pairs of individual assessments. We propose a sequential tie-breaking process and provide some properties of the degrees of consensus.

We have also devised an agglomerative hierarchical clustering procedure where agents are grouped into clusters by defining the similarity between two groups of agents with respect to a subset of alternatives as the degree of consensus in the merged group. We have illustrated our proposal from the qualitative marks obtained by a group of students in several subjects.

The rest of the paper is organized as follows. Section 2 is devoted to introduce and analyze ordinal proximity measures. In Section 3 we propose some applications to consensus and clustering. And Section 4 includes some concluding remarks.

\section{Ordinal proximity measures}

Let $A=\{1, \ldots, m\}$, with $m \geq 2$, be a set of agents and let $X=\left\{x_{1}, \ldots, x_{n}\right\}$, with $n \geq 2$, be the set of alternatives which have to be evaluated. Each agent assigns a linguistic term to every alternative within a finite linguistic ordered scale $\mathcal{L}=\left\{l_{1}, \ldots, l_{g}\right\}$, arranged from the lowest to the highest terms ${ }^{4}$, where the granularity of $\mathcal{L}$ is at least $3(g \geq 3)$.

\subsection{The model}

Consider that the psychological proximity between $l_{r} \in \mathcal{L}$ and $l_{s} \in \mathcal{L}$ is represented by $\pi_{r s}$ and let $\Delta=\left\{\pi_{r s} \mid r, s \in\{1, \ldots, g\}\right\}$ be the set of all possible psychological proximities between linguistic terms ${ }^{5}$.

Although we do not associate numbers to psychological proximities, we assume that it is possible to compare psychological proximities between linguistic terms through an asymmetric and transitive binary relation $\succ$ on $\Delta$, where $\pi_{r s} \succ \pi_{t u}$ means that the psychological proximity between $l_{r}$ and $l_{s}$ is bigger than the psychological proximity between $l_{t}$ and $l_{u}$.

We consider that the following properties should be satisfied for all $r, s, t, u \in$ $\{1, \ldots, g\}$ :

\footnotetext{
${ }^{4}$ For instance, Balinski and Laraki [4] consider the following six linguistic terms: 'to reject' $\left(l_{1}\right)$, 'poor' $\left(l_{2}\right)$, 'acceptable' $\left(l_{3}\right)$, 'good' $\left(l_{4}\right)$, 'very good' $\left(l_{5}\right)$ and 'excellent' $\left(l_{6}\right)$.

${ }^{5}$ At this stage we do not specify what kind of mathematical objects represent psychological proximities.
} 
1. If neither $\pi_{r s} \succ \pi_{t u}$ nor $\pi_{t u} \succ \pi_{r s}$, then $\pi_{r s}=\pi_{t u}$.

2. $\pi_{s r}=\pi_{r s}$.

3. $\pi_{r r}=\pi_{s s}$.

4. If $s \neq t$, then $\pi_{r r} \succ \pi_{s t}$.

5. If $r<s<t$, then $\pi_{r s} \succ \pi_{r t}$ and $\pi_{s t} \succ \pi_{r t}$.

6. If $r<s$ and $(r, s) \neq(1, g)$, then $\pi_{r s} \succ \pi_{1 g}$.

We now introduce a formal notion of proximity between linguistic terms with values on a finite chain (linear order) $\Delta=\left\{\delta_{1}, \ldots, \delta_{h}\right\}$, with $\delta_{1} \succ \cdots \succ \delta_{h}$, that captures the properties introduced above. The elements of $\Delta$ have no meaning and they only represent different degrees or proximity, being $\delta_{1}$ and $\delta_{h}$ the maximum and minimum degrees of proximity, respectively.

As usual in the setting of linear orders, $\delta_{r} \prec \delta_{s}$ means $\delta_{s} \succ \delta_{r} ; \delta_{r} \preceq \delta_{s}$ means $\delta_{r} \prec \delta_{s}$ or $\delta_{r}=\delta_{s}$; and $\delta_{r} \succeq \delta_{s}$ means $\delta_{r} \succ \delta_{s}$ or $\delta_{r}=\delta_{s}$.

First we assume that all the elements of $\Delta$ are relevant because they are reached as the degree of proximity between at least a pair of linguistic terms (exhaustiveness). We also assume that the proximity between a pair of linguistic terms does not depend on the order these terms are presented (symmetry), and the maximum proximity between linguistic terms is only reached when comparing a term with itself. Additionally, we assume that, given three different linguistic terms, the degree of proximity between the lowest and the highest terms should be smaller than the degrees of proximity between the lowest and the intermediate terms and also between the intermediate and the highest terms (monotonicity).

Definition 1. An ordinal proximity measure on $\mathcal{L}$ with values in $\Delta$ is a mapping $\pi: \mathcal{L}^{2} \longrightarrow \Delta$, where $\pi\left(l_{r}, l_{s}\right)=\pi_{r s}$ means the degree of proximity between $l_{r}$ and $l_{s}$, satisfying the following conditions:

1. Exhaustiveness: For every $\delta \in \Delta$, there exist $l_{r}, l_{s} \in \mathcal{L}$ such that $\delta=\pi_{r s}$.

2. Symmetry: $\pi_{s r}=\pi_{r s}$, for all $r, s \in\{1, \ldots, g\}$.

3. Maximum proximity: $\pi_{r s}=\delta_{1} \Leftrightarrow r=s$, for all $r, s \in\{1, \ldots, g\}$.

4. Monotonicity: $\min \left\{\pi_{r s}, \pi_{s t}\right\} \succ \pi_{r t}$, for all $r, s, t \in\{1, \ldots, g\}$ such that $r<s<t$.

Every ordinal proximity measure can be represented by a $g \times g$ symmetric matrix with coefficients in $\Delta$, where the elements in the main diagonal are $\pi_{r r}=\delta_{1}, r=1, \ldots, g$ :

$$
\left(\begin{array}{ccccc}
\pi_{11} & \cdots & \pi_{1 s} & \cdots & \pi_{1 g} \\
\cdots & \cdots & \cdots & \cdots & \cdots \\
\pi_{r 1} & \cdots & \pi_{r s} & \cdots & \pi_{r g} \\
\cdots & \cdots & \cdots & \cdots & \cdots \\
\pi_{g 1} & \cdots & \pi_{g s} & \cdots & \pi_{g g}
\end{array}\right)=\left(\pi_{r s}\right) .
$$

This matrix is called proximity matrix. 
Taking into account the conditions of Definition 1, it is only necessary to show the upper half proximity matrix

$$
\left(\begin{array}{cccccc}
\delta_{1} & \pi_{12} & \pi_{13} & \cdots & \pi_{1(g-1)} & \pi_{1 g} \\
& \delta_{1} & \pi_{23} & \cdots & \pi_{2(g-1)} & \pi_{2 g} \\
& & & \cdots & \cdots & \cdots \\
& & & & \delta_{1} & \pi_{(g-1) g} \\
& & & & & \delta_{1}
\end{array}\right) .
$$

Proposition 1. The four conditions appearing in Definition 1 are independent.

\section{PROOF:}

1. The matrix

$$
\left(\begin{array}{lll}
\delta_{1} & \delta_{2} & \delta_{3} \\
\delta_{2} & \delta_{1} & \delta_{3} \\
\delta_{3} & \delta_{3} & \delta_{1}
\end{array}\right)
$$

satisfies conditions 1,2 and 3 , but not condition $4: \min \left\{\pi_{12}, \pi_{23}\right\}=\delta_{3}=$ $\pi_{13}$.

2. The matrix

$$
\left(\begin{array}{lll}
\delta_{1} & \delta_{3} & \delta_{4} \\
\delta_{3} & \delta_{1} & \delta_{3} \\
\delta_{4} & \delta_{3} & \delta_{2}
\end{array}\right)
$$

satisfies conditions 1,2 and 4, but not condition $3: \pi_{33}=\delta_{2} \neq \delta_{1}$.

3. The matrix

$$
\left(\begin{array}{lll}
\delta_{1} & \delta_{2} & \delta_{4} \\
\delta_{3} & \delta_{1} & \delta_{2} \\
\delta_{4} & \delta_{2} & \delta_{1}
\end{array}\right)
$$

satisfies conditions 1,3 and 4 , but not condition 2: $\pi_{12}=\delta_{2} \neq \delta_{3}=\pi_{21}$.

4. The matrix

$$
\left(\begin{array}{lll}
\delta_{1} & \delta_{2} & \delta_{4} \\
\delta_{2} & \delta_{1} & \delta_{2} \\
\delta_{4} & \delta_{2} & \delta_{1}
\end{array}\right)
$$

satisfies conditions 2, 3 and 4 , but not condition $1: \delta_{3} \neq \pi_{r s}$ for all $r, s \in\{1, \ldots, g\}$.

\subsection{Some results}

In the following proposition we establish that the minimum proximity between linguistic terms is only reached when comparing the extreme linguistic terms $l_{1}$ and $l_{g}$.

Proposition 2. For all $r, s \in\{1, \ldots, g\}, \pi_{r s}=\delta_{h} \Leftrightarrow(r, s) \in\{(1, g),(g, 1)\}$. 
Proof: By way of contradiction, suppose there exist $r, s \in\{1, \ldots, g\}$ such that $r<s,(r, s) \neq(1, g)$ and $\pi_{r s}=\delta_{h}$. If $s<g$, by $\min \left\{\pi_{r s}, \pi_{s g}\right\} \succ \pi_{r g}$, we have $\delta_{h} \succ \pi_{r g}$, that is a contradiction. If $s=g$, then $1<r<g$. By $\min \left\{\pi_{1 r}, \pi_{r g}\right\} \succ \pi_{1 g}$, we have $\delta_{h} \succ \pi_{1 g}$, that is a contradiction.

In the following proposition we extend monotonicity to four linguistic terms (similar extensions can be made for more than four linguistic terms).

Proposition 3. For all $r, s, t, u \in\{1, \ldots, g\}$ such that $r<s<t<u$, the following properties hold:

1. If $\pi_{r s} \succ \pi_{s t}$, then $\pi_{r s} \succ \pi_{s u}$.

2. If $\pi_{r s} \succ \pi_{t u}$, then $\pi_{r s} \succ \pi_{s u}$.

3. If $\pi_{t u} \succ \pi_{s t}$, then $\pi_{t u} \succ \pi_{r t}$.

4. If $\pi_{t u} \succ \pi_{r s}$, then $\pi_{t u} \succ \pi_{r t}$.

5. If $\pi_{r t} \succ \pi_{t u}$, then $\pi_{r t} \succ \pi_{s u}$.

6. If $\pi_{s u} \succ \pi_{r s}$, then $\pi_{s u} \succ \pi_{r t}$.

\section{ProOF:}

1. Since $\min \left\{\pi_{s t}, \pi_{t u}\right\} \succ \pi_{s u}$, we have $\pi_{s t} \succ \pi_{s u}$. By $\pi_{r s} \succ \pi_{s t}$ and transitivity of $\succ$, we obtain $\pi_{r s} \succ \pi_{s u}$.

2. Since $\min \left\{\pi_{s t}, \pi_{t u}\right\} \succ \pi_{s u}$, we have $\pi_{t u} \succ \pi_{s u}$. By $\pi_{r s} \succ \pi_{t u}$ and transitivity of $\succ$, we obtain $\pi_{r s} \succ \pi_{s u}$.

3. Since $\min \left\{\pi_{r s}, \pi_{s t}\right\} \succ \pi_{r t}$, we have $\pi_{s t} \succ \pi_{r t}$. By $\pi_{t u} \succ \pi_{s t}$ and transitivity of $\succ$, we obtain $\pi_{t u} \succ \pi_{r t}$.

4. Since $\min \left\{\pi_{r s}, \pi_{s t}\right\} \succ \pi_{r t}$, we have $\pi_{r s} \succ \pi_{r t}$. By $\pi_{t u} \succ \pi_{r s}$ and transitivity of $\succ$, we obtain $\pi_{t u} \succ \pi_{r t}$.

5. Since $\min \left\{\pi_{s t}, \pi_{t u}\right\} \succ \pi_{s u}$, we have $\pi_{t u} \succ \pi_{s u}$. By $\pi_{r t} \succ \pi_{t u}$ and transitivity of $\succ$, we obtain $\pi_{r t} \succ \pi_{s u}$.

6. Since $\min \left\{\pi_{r s}, \pi_{s t}\right\} \succ \pi_{r t}$, we have $\pi_{r s} \succ \pi_{r t}$. By $\pi_{s u} \succ \pi_{r s}$ and transitivity of $\succ$, we obtain $\pi_{s u} \succ \pi_{r t}$.

In the following result, we show that the cardinality of the chain $\Delta$ is located between the granularity of $\mathcal{L}$ and a polinomial of degree 2 of that granularity.

Proposition 4. The following condition holds:

$$
g \leq h \leq \frac{g \cdot(g-1)}{2}+1 .
$$

Proof: The minimum cardinality of $\Delta$ is obtained if the proximities $\pi_{r s}$ are identical whenever $|s-r|$ is fixed. Since $|s-r| \in\{0,1, \ldots, g-1\}$, the minimum value of $h$ is $g$. 
The maximum number of degrees of proximity (without considering $\delta_{1}$ and $\left.\delta_{h}\right)$ are shown by rows

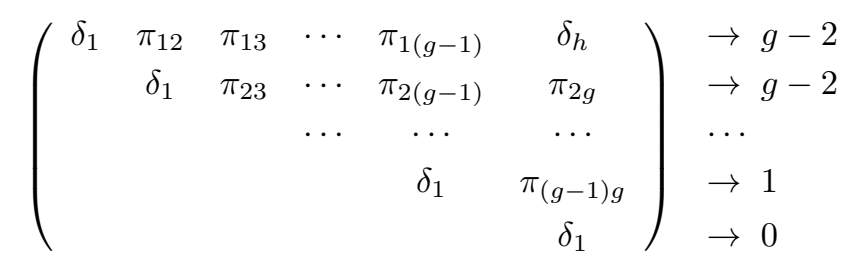

i.e., it is

$$
(g-1)-1+(g-2)+\cdots+1=\frac{g \cdot(g-1)}{2}-1 .
$$

Taking into account the two extreme degrees of proximity $\delta_{1}$ and $\delta_{h}$, the maximum value of $h$ is

$$
2+\frac{g \cdot(g-1)}{2}-1=\frac{g \cdot(g-1)}{2}+1 .
$$

Table 1 shows how the cardinality of $\Delta$ varies according to the granularity of $\mathcal{L}$, for $g=3,4, \ldots, 11$.

\begin{tabular}{cc}
$g$ & Cardinality of $\Delta$ \\
\hline 3 & $3 \leq h \leq 4$ \\
4 & $4 \leq h \leq 7$ \\
5 & $5 \leq h \leq 11$ \\
6 & $6 \leq h \leq 16$ \\
7 & $7 \leq h \leq 22$ \\
8 & $8 \leq h \leq 29$ \\
9 & $9 \leq h \leq 37$ \\
10 & $10 \leq h \leq 46$ \\
11 & $11 \leq h \leq 56$
\end{tabular}

Table 1: Cardinality of $\Delta$ according to the granularity of $\mathcal{L}$.

\subsection{Generating ordinal proximity measures from pairwise comparisons}

In the following example, we show what are the possible chains $\Delta$ and ordinal proximity measures $\pi$ in the simplest situation of a scale formed by three linguistic terms.

Example 1. For $g=3$, two cases of $\Delta$ and three different ordinal proximity measures $\pi$ are possible:

1. The balanced case, where $\pi_{12}=\pi_{23}=\delta_{2}$ 


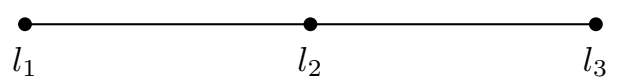

and the proximity matrix

$$
\left(\begin{array}{lll}
\delta_{1} & \delta_{2} & \delta_{3} \\
\delta_{2} & \delta_{1} & \delta_{2} \\
\delta_{3} & \delta_{2} & \delta_{1}
\end{array}\right)
$$

2. The unbalanced case with $\pi_{23}=\delta_{2} \succ \delta_{3}=\pi_{12}$

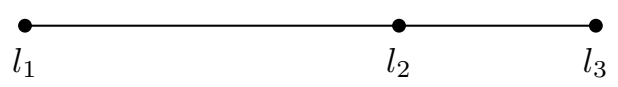

and the proximity matrix

$$
\left(\begin{array}{lll}
\delta_{1} & \delta_{3} & \delta_{4} \\
\delta_{3} & \delta_{1} & \delta_{2} \\
\delta_{4} & \delta_{2} & \delta_{1}
\end{array}\right)
$$

3. The unbalanced case with $\pi_{12}=\delta_{2} \succ \delta_{3}=\pi_{23}$

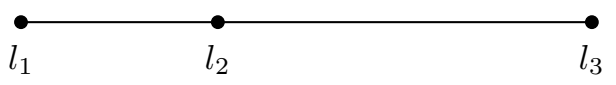

and the proximity matrix

$$
\left(\begin{array}{lll}
\delta_{1} & \delta_{2} & \delta_{4} \\
\delta_{2} & \delta_{1} & \delta_{3} \\
\delta_{4} & \delta_{3} & \delta_{1}
\end{array}\right)
$$

It is easy to check that all the previous matrices satisfy the conditions appearing in Definition 1.

Notice that in the balanced case $h=3$, but in the unbalanced cases $h=4$.

For $g>3$ the complexity increases, as we can see in the following remark.

Remark 1. The $g \times g$ proximity matrix $\left(\pi_{r s}\right)$ contains $g^{2}$ degrees of proximity. If we compare by pairs all these degrees of proximity, we would have to make $\left(g^{2}\right)^{2}=g^{4}$ comparisons. However, some of them are not necessary.

By Definition 1, we know that $\pi_{r r}=\delta_{1} \succ \pi_{r s}$ for all $r, s \in\{1, \ldots, g\}$ such that $r \neq s ; \pi_{s r}=\pi_{r s}$ for all $r, s \in\{1, \ldots, g\}$; and $\pi_{r s} \succ \pi_{r t}$ and $\pi_{s t} \succ \pi_{r t}$ for all $r, s, t \in\{1, \ldots, g\}$ such that $r<s<t$. By Proposition 2, we also know that $\pi_{r s} \succ \pi_{1 g}=\delta_{h}$ for all $r, s \in\{1, \ldots, g\}$ such that $(r, s) \notin\{(1, g),(g, 1)\}$. And by Proposition 3, if $r<s<t<u$ and $\pi_{r s} \succ \pi_{s t}$, then $\pi_{r s} \succ \pi_{s u}$; etc.

Consider now the case of $g=4$, where we only need to compare by pairs the following five degrees of proximity: $\pi_{12}, \pi_{13}, \pi_{23}, \pi_{24}$ and $\pi_{34}$. 
Notice that, by Definition 1, we know that $\pi_{12} \succ \pi_{13}, \pi_{23} \succ \pi_{13}, \pi_{23} \succ \pi_{24}$ and $\pi_{34} \succ \pi_{24}$. Then, we have the following pairwise comparisons:

$$
\begin{array}{cccc}
\pi_{12} \succ \pi_{13} & \pi_{12}-\pi_{23} & \pi_{12}-\pi_{24} & \pi_{12}-\pi_{34} \\
& \pi_{13} \prec \pi_{23} & \pi_{13}-\pi_{24} & \pi_{13}-\pi_{34} \\
& & \pi_{23} \succ \pi_{24} & \pi_{23}-\pi_{34} \\
& & & \pi_{24} \prec \pi_{34}
\end{array}
$$

Additionally, by Proposition 3, we also know the following implications:

$$
\begin{aligned}
& \pi_{12} \succ \pi_{23} \Rightarrow \pi_{12} \succ \pi_{24} \\
& \pi_{12} \succ \pi_{34} \Rightarrow \pi_{12} \succ \pi_{24} \\
& \pi_{34} \succ \pi_{23} \Rightarrow \pi_{34} \succ \pi_{13} \\
& \pi_{34} \succ \pi_{12} \Rightarrow \pi_{34} \succ \pi_{13} \\
& \pi_{13} \succ \pi_{34} \Rightarrow \pi_{13} \succ \pi_{24} \\
& \pi_{24} \succ \pi_{12} \Rightarrow \pi_{24} \succ \pi_{13} .
\end{aligned}
$$

Consequently, there are three essential pairwise comparisons: $\pi_{12}-\pi_{23}$, $\pi_{12}-\pi_{34}$ and $\pi_{23}-\pi_{34}$. Depending on the results of these comparisons, the following comparisons could be also necessary: $\pi_{12}-\pi_{24}, \pi_{13}-\pi_{34}$ and $\pi_{13}-\pi_{24}$. Thus, among the $16^{2}=256$ potential pairwise comparisons $\left(\pi_{r s}\right.$ versus $\pi_{t u}$ for all $r, s, t, u \in\{1, \ldots, 4\}$ ), only between three and six pairwise comparisons would be needed.

Following the pattern introduced in Remark 1 for $g=4$, it is possible to determine the proximity matrix for $g>4$ from some basic pairwise comparisons.

Remark 2. Given a finite linguistic ordered scale $\mathcal{L}=\left\{l_{1}, \ldots, l_{g}\right\}$, an important issue is who determines the proximity matrix. A possibility is that a representative group of agents $B$ declare how they understand the proximities between the basic pairs of linguistic terms, and then a majority rule is applied for determining $\Delta$ and the corresponding degrees of proximity.

Simple majority is the most decisive majority (when indifferences are allowed, the winner can have very poor support). Qualified majorities require more support for declaring a winner and they go from absolute majority to unanimity. In turn, majorities based on difference of votes go from simple majority to unanimity (see García-Lapresta and Llamazares [18], Llamazares [35] and Llamazares et al. [36]). Figure 1 illustrates the relationships among the mentioned majorities.

We now introduce a method for generating social proximity outcomes, through different majorities, from the pairwise comparisons among degrees of proximity for all the basic pairs $\left(l_{r}, l_{s}\right),\left(l_{t}, l_{u}\right) \in \mathcal{L}^{2}$. We have considered that the statu $q u o$ is $\pi_{r s}=\pi_{t u}$ : this should be the outcome whenever there does not exist a majority, previously fixed, declaring $\pi_{r s} \succ \pi_{t u}$ or $\pi_{t u} \succ \pi_{r s}$.

With $\pi_{r s} \succ_{b} \pi_{t u}$ we denote that agent $b \in B$ declares that $l_{r}$ is closer to $l_{s}$ than $l_{t}$ is to $l_{u}$. Analogously, $\pi_{r s}={ }_{b} \pi_{t u}$ denotes that agent $b \in B$ declares that the proximity between $l_{s}$ and $l_{t}$ is the same as that between $l_{t}$ and $l_{u}$. 


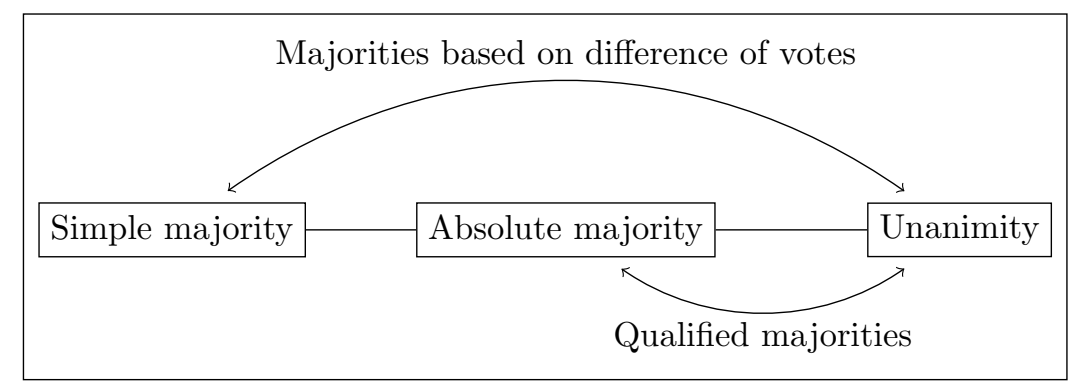

Figure 1: Majorities.

1. If the majority based on difference of $k \in\{0,1, \ldots, \# B-1\}$ votes is applied $^{6}$ :

(a) $\pi_{r s} \succ \pi_{t u} \Leftrightarrow\left(\#\left\{b \in B \mid \pi_{r s} \succ_{b} \pi_{t u}\right\}>\#\left\{b \in B \mid \pi_{t u} \succ_{b} \pi_{r s}\right\}+k\right.$ and $\left.\#\left\{b \in B \mid \pi_{r s} \succ_{b} \pi_{t u}\right\}>\#\left\{b \in B \mid \pi_{r s}={ }_{b} \pi_{t u}\right\}+k\right)$,

(b) $\pi_{t u} \succ \pi_{r s} \Leftrightarrow\left(\#\left\{b \in B \mid \pi_{t u} \succ_{b} \pi_{r s}\right\}>\#\left\{b \in B \mid \pi_{r s} \succ_{b} \pi_{t u}\right\}+k\right.$ and $\left.\#\left\{b \in B \mid \pi_{t u} \succ_{b} \pi_{r s}\right\}>\#\left\{b \in B \mid \pi_{r s}={ }_{b} \pi_{t u}\right\}+k\right)$,

(c) $\pi_{r s}=\pi_{t u}$, otherwise.

2. If the qualified majority of threshold $q \in[0.5,1)$ is applied ${ }^{7}$ :

(a) $\pi_{r s} \succ \pi_{t u} \Leftrightarrow \#\left\{b \in B \mid \pi_{r s} \succ_{b} \pi_{t u}\right\}>q \cdot \# B$,

(b) $\pi_{t u} \succ \pi_{r s} \Leftrightarrow \#\left\{b \in B \mid \pi_{t u} \succ_{b} \pi_{r s}\right\}>q \cdot \# B$,

(c) $\pi_{r s}=\pi_{t u}$, otherwise.

Once this information has been collected, it is necessary to adjust the ordinal information for providing the chain $\Delta$ and the proximity matrix satisfying the conditions appearing in Definition 1, as in Remark 1.

It is important noticing that the mentioned majorities may generate intransitivities in $\succ$ or in $=$, i.e., there can exist linguistic terms $l_{r}, l_{s}, l_{t}, l_{u}, l_{v}, l_{w} \in \mathcal{L}$ such that, after aggregating the individual opinions by a specific majority, it occurs $\pi_{r s} \succ \pi_{t u}, \pi_{t u} \succ \pi_{v w}$ and not $\pi_{r s} \succ \pi_{v w}$ (either $\pi_{v w} \succ \pi_{r s}$ or $\left.\pi_{v w}=\pi_{r s}\right)$; or $\pi_{r s}=\pi_{t u}, \pi_{t u}=\pi_{v w}$ and $\pi_{r s} \neq \pi_{v w}$ (either $\pi_{r s} \succ \pi_{v w}$ or $\pi_{v w} \succ \pi_{r s}$ ). Monotonicity and Proposition 3 can also be violated after the aggregation process. These kinds of paradoxes and inconsistencies may be considered as specific problems of judgment aggregation theory within the social choice theory (see Dietrich and List [14], List [34], Mongin [38] and Grossi and Pigozzi [23], among others).

When some of the above inconsistencies appear, a decision maker would select an appropriate chain $\Delta$ and the corresponding proximity matrix. Obviously, another possibility would be for that group of agents to vote on their preferred

\footnotetext{
${ }^{6}$ Simple majority corresponds to the case $k=0$.

${ }^{7}$ Absolute majority corresponds to the case $q=0.5$.
} 
proximity matrices and then apply an appropriate voting system for obtaining the outcome. This avoids the appearance of inconsistencies but agents have to analyze a high number of complex alternatives.

\subsection{A field experiment}

We now present the results of a survey made to 76 members of the Spanish Society for Fuzzy Logic and Technology (ESTYLF) about the degrees of proximity among the usual decisions of some journal editors (see Table 2).

\begin{tabular}{cccc}
$l_{1}$ & $l_{2}$ & $l_{3}$ & $l_{4}$ \\
\hline Reject & Major revision & Minor revision & Accept \\
& & \\
& &
\end{tabular}

The data obtained in the survey are included in Table 3.

\begin{tabular}{ccc}
$\pi_{12}$ versus $\pi_{23}$ & Number & $\%$ \\
\hline$\pi_{12} \succ \pi_{23}$ & 27 & 35.5 \\
$\pi_{12} \prec \pi_{23}$ & 32 & 42.1 \\
$\pi_{12}=\pi_{23}$ & 17 & 22.4
\end{tabular}

\begin{tabular}{ccc}
$\pi_{23}$ versus $\pi_{34}$ & Number & $\%$ \\
\hline$\pi_{23} \succ \pi_{34}$ & 6 & 7.9 \\
$\pi_{23} \prec \pi_{34}$ & 69 & 90.8 \\
$\pi_{23}=\pi_{34}$ & 1 & 1.3
\end{tabular}

\begin{tabular}{ccc}
$\pi_{12}$ versus $\pi_{34}$ & Number & $\%$ \\
\hline$\pi_{12} \succ \pi_{34}$ & 10 & 13.2 \\
$\pi_{12} \prec \pi_{34}$ & 54 & 71.0 \\
$\pi_{12}=\pi_{34}$ & 12 & 15.8
\end{tabular}

\begin{tabular}{ccc}
$\pi_{12}$ versus $\pi_{24}$ & Number & $\%$ \\
\hline$\pi_{12} \succ \pi_{24}$ & 42 & 55.3 \\
$\pi_{12} \prec \pi_{24}$ & 18 & 23.7 \\
$\pi_{12}=\pi_{24}$ & 16 & 21.0
\end{tabular}

\begin{tabular}{ccc}
$\pi_{13}$ versus $\pi_{34}$ & Number & $\%$ \\
\hline$\pi_{13} \succ \pi_{34}$ & 0 & 0.0 \\
$\pi_{13} \prec \pi_{34}$ & 75 & 98.7 \\
$\pi_{13}=\pi_{34}$ & 1 & 1.3
\end{tabular}

\begin{tabular}{ccc}
$\pi_{13}$ versus $\pi_{24}$ & Number & $\%$ \\
\hline$\pi_{13} \succ \pi_{24}$ & 1 & 1.3 \\
$\pi_{13} \prec \pi_{24}$ & 53 & 69.7 \\
$\pi_{13}=\pi_{24}$ & 22 & 29.0
\end{tabular}

Table 3: Data of the survey.

We now present the results generated by simple and absolute majorities when aggregating individual opinions of the proximities between linguistic terms.

- Simple majority:

$\pi_{34} \succ \pi_{23} \succ \pi_{12} \succ \pi_{24} \succ \pi_{13} \succ \pi_{14}$ and $\pi_{r r}=\delta_{1}$ for every $r \in$ $\{1, \ldots, 4\}$.

This information allows us to assign the following degrees of proximity

$$
\pi_{r r}=\delta_{1} \succ \pi_{34}=\delta_{2} \succ \pi_{23}=\delta_{3} \succ \pi_{12}=\delta_{4} \succ \pi_{24}=\delta_{5} \succ \pi_{13}=\delta_{6} \succ \pi_{14}=\delta_{7}
$$


and the upper half proximity matrix

$$
\left(\begin{array}{cccc}
\delta_{1} & \delta_{4} & \delta_{6} & \delta_{7} \\
& \delta_{1} & \delta_{3} & \delta_{5} \\
& & \delta_{1} & \delta_{2} \\
& & & \delta_{1}
\end{array}\right)
$$

that can be visualized as follows

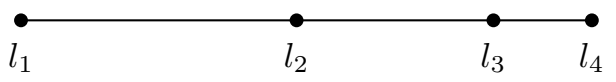

- Absolute majority:

$\pi_{34} \succ \pi_{12}=\pi_{23} \succ \pi_{24} \succ \pi_{13} \succ \pi_{14}$ and $\pi_{r r}=\delta_{1}$ for every $r \in\{1, \ldots, 4\}$.

This information allows us to assign the following degrees of proximity

$$
\pi_{r r}=\delta_{1} \succ \pi_{34}=\delta_{2} \succ \pi_{12}=\pi_{23}=\delta_{3} \succ \pi_{24}=\delta_{4} \succ \pi_{13}=\delta_{5} \succ \pi_{14}=\delta_{6}
$$

and the upper half proximity matrix

$$
\left(\begin{array}{cccc}
\delta_{1} & \delta_{3} & \delta_{5} & \delta_{6} \\
& \delta_{1} & \delta_{3} & \delta_{4} \\
& & \delta_{1} & \delta_{2} \\
& & & \delta_{1}
\end{array}\right)
$$

that can be visualized as follows

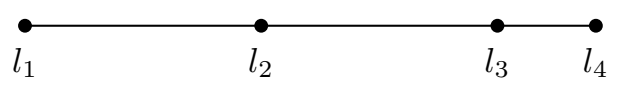

\section{Applications to consensus and clustering}

Once ordinal proximity measures have been introduced and analyzed, we now propose some applications to consensus and clustering.

\subsection{Consensus}

For measuring the degree of consensus among a group of agents that provide their opinions on a set of alternatives, different proposals can be found in the literature (see Martínez-Panero [37] for an overview of different notions of consensus).

In the fuzzy framework, there exists a huge amount of literature where some degrees of consensus have been defined (see Spillman et al. [42], Kacprzyk and Fedrizzi [28, 29, 30], Kacprzyk et al. [31], Fedrizzi et al. [17], Herrera et al. [25] 
and Bordogna et al. [6], among others). Some referenced surveys can be found in Cabrerizo et al. [10], Herrera-Viedma et al. [26] and Palomares et al. [39].

In the social choice framework, the notion of consensus measure was introduced by Bosch [8] in the context of linear orders. Additionally, Bosch [8] and Alcalde-Unzu and Vorsatz [1] provided axiomatic characterizations of several consensus measures in the context of linear orders. García-Lapresta and Pérez-Román [19] extended that notion to the context of weak orders and they analyzed a class of consensus measures generated by distances. Alcantud et al. [2] provided axiomatic characterizations of some consensus measures in the setting of approval voting. In turn, Erdamar et al. [15] extended the notion of consensus measure to the preference-approval setting through different kinds of distances, and García-Lapresta et al. [22] introduced another extension to the framework of hesitant linguistic assessments.

A profile is a matrix

$$
V=\left(\begin{array}{ccccc}
v_{1}^{1} & \cdots & v_{i}^{1} & \cdots & v_{n}^{1} \\
\cdots & \cdots & \cdots & \cdots & \cdots \\
v_{1}^{a} & \cdots & v_{i}^{a} & \cdots & v_{n}^{a} \\
\cdots & \cdots & \cdots & \cdots & \cdots \\
v_{1}^{m} & \cdots & v_{i}^{m} & \cdots & v_{n}^{m}
\end{array}\right)=\left(v_{i}^{a}\right)
$$

consisting of $m$ rows and $n$ columns of linguistic terms, where the element $v_{i}^{a} \in \mathcal{L}$ represents the linguistic assessment given by the agent $a \in A$ to the alternative $x_{i} \in X$.

With $\# I$ we denote the cardinality of $I$. With $\mathcal{P}_{2}(A)=\{I \subseteq A \mid \# I \geq 2\}$ we denote the family of subsets of at least two agents.

For measuring the consensus in a group of agents over a set of alternatives, we start ordering all the degrees of proximity between individual assessments over the alternatives in a decreasing fashion, i.e, from highest to lowest degrees of proximity. Then, we define the degree of consensus as the upper median of the degrees of proximity.

We have to note that when the number of elements in a list of ordered elements is odd, the median is unique. But if that number is even, then there are two medians; in that case, we choose the upper median (in our context, the lowest degree of proximity, i.e., the pessimistic outcome), as proposed by Balinski and Laraki in the voting system Majority Judgment $[3,4]$ for individual linguistic assessments (they consider the lower median because they arrange the inputs in an increasing fashion).

Definition 2. Given a profile $V=\left(v_{i}^{a}\right)$, the degree of consensus in a subset of agents $I \in \mathcal{P}_{2}(A)$ over a subset of alternatives $\emptyset \neq Y \subseteq X$ is defined as

$$
C(I, Y)=M\left(\pi\left(v_{i}^{a}, v_{i}^{b}\right)_{\substack{a, b \in I, a<b \\ x_{i} \in Y}}\right) \in \Delta,
$$

where $M$ is the upper median operator. 
Remark 3. For each alternative, the number of pairwise comparisons between individual assessments given by the agents of $I$ is

$$
p(I)=\frac{\# I \cdot(\# I-1)}{2} .
$$

In the following proposition we show that the degree of consensus is not susceptible to be considered either too high or too low.

Proposition 5. Given a profile $V=\left(v_{i}^{a}\right)$, for every subset of agents $I \in$ $\mathcal{P}_{2}(A)$ and every subset of alternatives $\emptyset \neq Y \subseteq X$, the following conditions hold:

$$
\#\left\{\left(a, b, x_{i}\right) \in I \times I \times Y \mid a<b, \pi\left(v_{i}^{a}, v_{i}^{b}\right) \succeq C(I, Y)\right\} \geq \frac{p(I) \cdot \# Y}{2}
$$

and

$$
\#\left\{\left(a, b, x_{i}\right) \in I \times I \times Y \mid a<b, \pi\left(v_{i}^{a}, v_{i}^{b}\right) \preceq C(I, Y)\right\} \geq \frac{p(I) \cdot \# Y}{2} .
$$

Proof: It is a direct consequence of the notion of medians.

In the following proposition, we provide necessary and sufficient conditions for having maximum and minimum degrees of consensus (in the last case only for two agents and one alternative).

Proposition 6. Given a profile $V=\left(v_{i}^{a}\right)$, a subset of agents $I \in \mathcal{P}_{2}(A)$, two agents $a, b \in A$, a subset of alternatives $\emptyset \neq Y \subseteq X$ and an alternative $x_{i} \in X$, the following properties hold:

1. $C(I, Y)=\delta_{1}$ if and only if there exists $r \in\{1, \ldots, g\}$ such that

$$
\#\left\{\left(a, x_{i}\right) \in I \times Y \mid v_{i}^{a}=l_{r}\right\}>\frac{p(I) \cdot \# Y}{2} .
$$

2. $C\left(\{a, b\},\left\{x_{i}\right\}\right)=\delta_{h}$ if and only if $\left(v_{i}^{a}, v_{i}^{b}\right) \in\left\{\left(l_{1}, l_{g}\right),\left(l_{g}, l_{1}\right)\right\}$.

\section{ProOF:}

1. First, notice that the number of elements in the list $\pi\left(v_{i}^{a}, v_{i}^{b}\right)_{\substack{a, b \in I, a<b \\ x_{i} \in Y}}$ is $p(I) \cdot \# Y$. Since $C(I, Y)=\delta_{1}$ if and only the upper median of that list is $\delta_{1}$, i.e., more than a half of the assessments are the same, say $l_{r}$, we obtain the result.

2. It follows from Proposition 2.

Since the cardinality of $\Delta$ could be low, it is very easy to have ties among the degrees of consensus in different subsets of agents. We now introduce a sequential tie-breaking process based on one of the proposals of Balinski and Laraki [3]. 
Starting from $C^{(1)}(I, Y)=C(I, Y)$, we calculate $C^{(2)}(I, Y)$ as in $C(I, Y)$ but after dropping the upper median of the list $\pi\left(v_{i}^{a}, v_{i}^{b}\right)_{a, b \in I, a<b}$, and analogously for $C^{(2)}(I, Y)$, etc.

Definition 3. Given a profile $V=\left(v_{i}^{a}\right)$, the sequential consensus vector relative to a subset of agents $I \in \mathcal{P}_{2}(A)$ and a subset of alternatives $\emptyset \neq Y \subseteq X$ is defined as

$$
\boldsymbol{C}(I, Y)=\left(C^{(1)}(I, Y), C^{(2)}(I, Y), \ldots, C^{(p(I))}(I, Y)\right) \in \Delta^{p(I) \cdot \# Y} .
$$

Definition 4. Given a profile $V=\left(v_{i}^{a}\right)$, the consensus order relative to a subset of alternatives $\emptyset \neq Y \subseteq X$ is defined as

$$
I \succcurlyeq_{Y} J \text { if } \boldsymbol{C}(I, Y) \succcurlyeq_{\ell} \boldsymbol{C}(J, Y)
$$

where $\succcurlyeq_{\ell}$ is the lexicographic weak order on $\Delta^{\min \{p(I) \cdot \# Y, p(J) \cdot \# Y\}}$, i.e., $I \succcurlyeq_{Y}$ $J$ if one of the following conditions hold:

$$
\begin{aligned}
& C^{(1)}(I, Y) \succ C^{(1)}(J, Y) \\
& C^{(1)}(I, Y)=C^{(1)}(J, Y) \text { and } C^{(2)}(I, Y) \succ C^{(2)}(J, Y) \\
& C^{(1)}(I, Y)=C^{(1)}(J, Y), C^{(2)}(I, Y)=C^{(2)}(J, Y) \text { and } C^{(3)}(I, Y) \succ C^{(3)}(J, Y)
\end{aligned}
$$

3.2. Clustering

In many contexts and disciplines, observations or objects are grouped in clusters in such a way that elements within each cluster are similar to one another with respect to an attribute. Then, objects are classified in homogeneous clusters and the objects in a cluster are more similar to each other than they are to an object belonging to a different cluster (see Jain et al. [27] and Everitt et al. [16], among others).

We now devise an agglomerative hierarchical clustering procedure in the context of unbalanced qualitative scales. More specifically, we have analyzed how agents can be grouped into clusters when such agents rate alternatives by means of linguistic terms from a predetermined unbalanced qualitative scale.

In the cluster formation, we have considered that the similarity between two groups of agents with respect to a set of alternatives is the degree of consensus in the merged group, as in García-Lapresta and Pérez-Román [20, 21] (in other settings). That consensus is measured by taking into account the degrees of proximity between all the pairs of individual assessments over the alternatives that are being evaluated.

Definition 5. Given a profile $V=\left(v_{i}^{a}\right)$, the similarity function relative to a subset of alternatives $\emptyset \neq Y \subseteq X$

$$
S_{Y}:(\mathcal{P}(A) \backslash\{\emptyset\})^{2} \longrightarrow \Delta
$$


is defined as

$$
S_{Y}(I, J)= \begin{cases}C(I \cup J, Y), & \text { if } \#(I \cup J) \geq 2, \\ \delta_{1}, & \text { if } \#(I \cup J)=1 .\end{cases}
$$

Remark 4. Similarity and proximity are related notions. In this paper, the concept of ordinal proximity is applied to pairs of linguistic terms, while the concept of similarity is applied to pairs of subsets of agents (with respect a subset of alternatives). In the extreme case of two agents and a single alternative, the similarity between these agents on that alternative is just the proximity between their assessments: given an alternative $x_{i} \in X$ and two different agents $a, b \in A$, we have

$$
S_{\left\{x_{i}\right\}}(\{a\},\{b\})=C\left(\{a, b\},\left\{x_{i}\right\}\right)=\pi\left(v_{i}^{a}, v_{i}^{b}\right) .
$$

Definition 6. Given a profile $V=\left(v_{i}^{a}\right)$, the sequential similarity vector relative to a subset of alternatives $\emptyset \neq Y \subseteq X$ for $I, J \in(\mathcal{P}(A) \backslash\{\emptyset\})$ is defined as

$$
\boldsymbol{S}_{Y}(I, J)=\left(S_{Y}^{(1)}(I, J), S_{Y}^{(2)}(I, J), \ldots, S_{Y}^{(p(I \cup J) \cdot \# Y)}(I, J)\right) \in \Delta^{p(I \cup J) \cdot \# Y},
$$

where

$$
S_{Y}^{(k)}(I, J)= \begin{cases}C^{(k)}(I \cup J, Y), & \text { if } \#(I \cup J) \geq 2, \\ \delta_{1}, & \text { if } \#(I \cup J)=1 .\end{cases}
$$

Given a profile $V=\left(v_{i}^{a}\right)$ and a subset of alternatives $\emptyset \neq Y \subseteq X$, we consider all the degrees of proximity $\pi\left(v_{i}^{a}, v_{i}^{b}\right)$, with $a, b \in A$ and $x_{i} \in Y$.

The agglomerative hierarchical clustering procedure we propose has some similarities to the one provided by García-Lapresta and Pérez-Román [21]. It consists of a sequential process addressed by the following stages:

1. The initial clustering is $\mathcal{A}_{0}^{Y}=\{\{1\}, \ldots,\{m\}\}$.

2. Calculate the similarities between all the pairs of agents, $S_{Y}(\{a\},\{b\})$ for all $a, b \in A$.

3. Select the two agents $a, b \in A$ that maximize $\boldsymbol{S}_{Y}$ in a lexicographic manner (taking into account the corresponding sequential similarity vectors) and construct the first cluster $A_{1}^{Y}=\{a, b\}$.

4. The new clustering is $\mathcal{A}_{1}^{Y}=\left(\mathcal{A}_{0}^{Y} \backslash\{\{a\},\{b\}\}\right) \cup\left\{A_{1}^{Y}\right\}$.

5. Calculate the similarities $S_{Y}\left(A_{1}^{Y},\{c\}\right)$ and take into account the previously computed similarities $S_{Y}(\{c\},\{d\})$, for all $\{c\},\{d\} \in \mathcal{A}_{1}^{Y}$.

6. Select the two elements of $\mathcal{A}_{1}^{Y}$ that maximize $\boldsymbol{S}_{Y}$ in a lexicographic manner and construct the second cluster $A_{2}^{i}$.

7. Proceed as in previous items until obtaining the next clustering $\mathcal{A}_{2}^{i}$.

The process continues in the same way until obtaining the last cluster, $\mathcal{A}_{m-1}^{Y}=\{A\}$. 


\subsection{An illustrative example}

In order to illustrate how the proposed agglomerative hierarchical clustering process works, we have considered the qualitative evaluations obtained for 169 students of the first year of the Degree in Commerce at the University of Valladolid during the academic year 2013-2014.

Table 4 contains the usual qualitative four marks in the Spanish education system.

\begin{tabular}{c|cccc} 
& $l_{1}$ & $l_{2}$ & $l_{3}$ & $l_{4}$ \\
\hline Spanish & Suspenso & Aprobado & Notable & Sobresaliente \\
English & Fail & Sufficient & Good & Excellent
\end{tabular}

Table 4: Marks.

The nine subjects appearing in Table 5 have been considered the agents ${ }^{8}$ and the alternatives are the students that have been evaluated.

\begin{tabular}{ll} 
Agent & Name \\
\hline 1 & Economic History of Trade \\
2 & Introduction to Economic Theory \\
3 & Basics of Law \\
4 & Business Organization \\
5 & Mathematics for Business \\
6 & Spanish and World Economy \\
7 & Basics of Financial Accounting \\
8 & Information Technology for Commercial Management I \\
9 & Introduction to Statistics
\end{tabular}

Table 5: Subjects.

A survey about the proximities among the terms of Table 4 was made by 36 teachers of the Degree in Commerce at the University of Valladolid. The data obtained in the survey are included in Table 6 .

We now present the results generated by simple majority when aggregating individual opinions of the proximities between linguistic terms:

$\pi_{12}=\pi_{34} \succ \pi_{23} \succ \pi_{13}=\pi_{24} \succ \pi_{14}$ and $\pi_{r r}=\delta_{1}$ for every $r \in\{1, \ldots, 4\}$. This information allows us to assign the following degrees of proximity

$$
\pi_{r r}=\delta_{1} \succ \pi_{12}=\pi_{34}=\delta_{2} \succ \pi_{23}=\delta_{3} \succ \pi_{13}=\pi_{24}=\delta_{4} \succ \pi_{14}=\delta_{5}
$$

and the corresponding upper half proximity matrix

\footnotetext{
${ }^{8}$ Although the agents are the teachers that evaluate the students in each subject, for simplicity we have identified subjects and teachers (some subjects have several teachers, but marks are unique for each student in each subject).
} 


\begin{tabular}{ccc}
$\pi_{12}$ versus $\pi_{23}$ & Number & $\%$ \\
\hline$\pi_{12} \succ \pi_{23}$ & 17 & 47.2 \\
$\pi_{12} \prec \pi_{23}$ & 4 & 11.1 \\
$\pi_{12}=\pi_{23}$ & 15 & 41.7
\end{tabular}

\begin{tabular}{ccc}
$\pi_{23}$ versus $\pi_{34}$ & Number & $\%$ \\
\hline$\pi_{23} \succ \pi_{34}$ & 6 & 16.7 \\
$\pi_{23} \prec \pi_{34}$ & 17 & 47.2 \\
$\pi_{23}=\pi_{34}$ & 13 & 36.1
\end{tabular}

\begin{tabular}{ccc}
$\pi_{12}$ versus $\pi_{34}$ & Number & $\%$ \\
\hline$\pi_{12} \succ \pi_{34}$ & 10 & 27.8 \\
$\pi_{12} \prec \pi_{34}$ & 9 & 25.0 \\
$\pi_{12}=\pi_{34}$ & 17 & 47.2
\end{tabular}

\begin{tabular}{ccc}
$\pi_{12}$ versus $\pi_{24}$ & Number & $\%$ \\
\hline$\pi_{12} \succ \pi_{24}$ & 26 & 72.2 \\
$\pi_{12} \prec \pi_{24}$ & 0 & 0.0 \\
$\pi_{12}=\pi_{24}$ & 10 & 27.8
\end{tabular}

\begin{tabular}{ccc}
$\pi_{13}$ versus $\pi_{34}$ & Number & $\%$ \\
\hline$\pi_{13} \succ \pi_{34}$ & 0 & 0.0 \\
$\pi_{13} \prec \pi_{34}$ & 34 & 94.4 \\
$\pi_{13}=\pi_{34}$ & 2 & 5.6
\end{tabular}

\begin{tabular}{ccc}
$\pi_{13}$ versus $\pi_{24}$ & Number & $\%$ \\
\hline$\pi_{13} \succ \pi_{24}$ & 1 & 2.8 \\
$\pi_{13} \prec \pi_{24}$ & 5 & 13.9 \\
$\pi_{13}=\pi_{24}$ & 30 & 83.3
\end{tabular}

Table 6: Data of the survey.

$$
\left(\begin{array}{cccc}
\delta_{1} & \delta_{2} & \delta_{4} & \delta_{5} \\
& \delta_{1} & \delta_{3} & \delta_{4} \\
& & \delta_{1} & \delta_{2} \\
& & & \delta_{1}
\end{array}\right)
$$

that can be visualized as follows

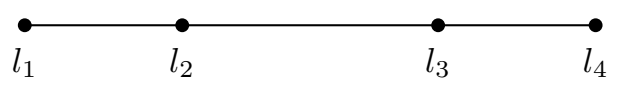

In order to show how the clustering process works, we have considered two cases. The computations for obtaining the corresponding sequential consensus and similarity vectors have been conducted with MATLAB.

1. The first profile we have considered is a matrix of 9 rows and 169 columns containing the 1521 marks obtained by the whole group of students in the 9 subjects. In this first clustering process, we have only focused in the total set of alternatives (students), i.e., $Y=X$.

After comparing the different sequential similarity vectors, we have obtained the following clusters of agents (subjects): 


$$
\begin{aligned}
& \mathcal{A}_{0}^{X}=\{\{1\},\{2\},\{3\},\{4\},\{5\},\{6\},\{7\},\{8\},\{9\}\} \\
& \mathcal{A}_{1}^{X}=\{\{5,7\},\{1\},\{2\},\{3\},\{4\},\{6\},\{8\},\{9\}\} \\
& \mathcal{A}_{2}^{X}=\{\{1,5,7\},\{2\},\{3\},\{4\},\{6\},\{8\},\{9\}\} \\
& \mathcal{A}_{3}^{X}=\{\{1,2,5,7\},\{3\},\{4\},\{6\},\{8\},\{9\}\} \\
& \mathcal{A}_{4}^{X}=\{\{1,2,3,5,7\},\{4\},\{6\},\{8\},\{9\}\} \\
& \mathcal{A}_{5}^{X}=\{\{1,2,3,4,5,7\},\{6\},\{8\},\{9\}\} \\
& \mathcal{A}_{6}^{X}=\{\{1,2,3,4,5,7,9\},\{6\},\{8\}\} \\
& \mathcal{A}_{7}^{X}=\{\{1,2,3,4,5,6,7,9\},\{8\}\} \\
& \mathcal{A}_{8}^{X}=\{\{1,2,3,4,5,6,7,8,9\}\} .
\end{aligned}
$$

This cluster formation can be interpreted as there does not exist a group structure in the set of agents (subjects). This is not unusual at all when the clustering is processed in an agglomerative hierarchical fashion.

2. The second profile we have considered is a matrix of 9 rows and 42 columns containing the 378 marks obtained by a group of 42 students in the 9 subjects.

Now we have obtained the following clusters of agents (subjects):

$$
\begin{aligned}
& \mathcal{A}_{0}^{X}=\{\{1\},\{2\},\{3\},\{4\},\{5\},\{6\},\{7\},\{8\},\{9\}\} \\
& \mathcal{A}_{1}^{X}=\{\{1,7\},\{2\},\{3\},\{4\},\{5\},\{6\},\{8\},\{9\}\} \\
& \mathcal{A}_{2}^{X}=\{\{1,7\},\{3,4\},\{2\},\{5\},\{6\},\{8\},\{9\}\} \\
& \mathcal{A}_{3}^{X}=\{\{1,7\},\{3,4,6\},\{2\},\{5\},\{8\},\{9\}\} \\
& \mathcal{A}_{4}^{X}=\{\{1,2,7\},\{3,4,6\},\{5\},\{8\},\{9\}\} \\
& \mathcal{A}_{5}^{X}=\{\{1,2,7\},\{3,4,6\},\{8,9\},\{5\}\} \\
& \mathcal{A}_{6}^{X}=\{\{1,2,5,7\},\{3,4,6\},\{8,9\}\} \\
& \mathcal{A}_{7}^{X}=\{\{1,2,5,7\},\{3,4,6,8,9\}\} \\
& \mathcal{A}_{8}^{X}=\{\{1,2,3,4,5,6,7,8,9\}\} .
\end{aligned}
$$

\section{Concluding remarks}

Using unbalanced qualitative scales requires some measurement of proximities among the terms of the scale. Since in this setting cardinal measurement of proximities is meaningless, we have proposed to make pairwise comparisons of proximities between pairs of terms within a purely ordinal approach. After these comparisons, it is possible to assign an element of an abstract chain to each proximity between pairs of linguistic terms. This ordinal approach allows to measure the degree of consensus in each group of agents over every subset of alternatives and, subsequently, to generate an agglomerative hierarchical clustering procedure on the set of agents which is based on the degrees of consensus in the possible merged groups of agents. 
As mentioned in Remark 2, an important and non-trivial issue is who and how to determine the chain $\Delta$ and the corresponding proximity matrix. When a group of agents declare their opinions on the ordinal proximities among the terms of an unbalanced qualitative scale and these opinions are aggregated through a voting system, inconsistencies may appear, as happens in different problems analyzed within the judgment aggregation theory. These aspects deserve special attention and they will be addressed in further research.

\section{Acknowledgments}

The authors are grateful to Luis Ángel García-Escudero, Bonifacio Llamazares, Reinhard Suck, Ali Ünlü and two anonymous referees for their comments and suggestions. The financial support of the Spanish Ministerio de Economía y Competitividad (project ECO2012-32178) and Consejería de Educación de la Junta de Castilla y León (project VA066U13) are acknowledged.

\section{References}

[1] Alcalde-Unzu, J., Vorsatz, M., Measuring the cohesiveness of preferences: an axiomatic analysis. Social Choice and Welfare 41, pp. 965-988, 2013.

[2] Alcantud, J.C.R., de Andrés, R., Cascón, J.M., On measures of cohesiveness under dichotomous opinions: some characterizations of Approval Consensus Measures. Information Sciences 240, pp. 45-55, 2013.

[3] Balinski, M., Laraki, R., A theory of measuring, electing and ranking. Proceedings of the National Academy of Sciences of the United States of America 104, pp. 8720-8725, 2007.

[4] Balinski, M., Laraki, R., Majority Judgment. Measuring, Ranking, and Electing. The MIT Press, Cambridge MA, 2011.

[5] Bennett, J.F., Hays, W.L., Multidimensional unfolding: Determining the dimensionality of ranked preference data. Psychometrika 25, pp. 27-43, 1960.

[6] Bordogna, G., Fedrizzi, M., Pasi, G., A linguistic modeling of consensus in group decision making based on OWA operators. IEEE Transactions on Systems, Man and Cybernetics, Part A: Systems and Humans 27, pp. 126-133, 1997.

[7] Borg, I., Groenen, P., Modern Multidimensional Scaling. Theory and Applications, 2nd edition. Springer, New York, 2005.

[8] Bosch, R., Characterizations of Voting Rules and Consensus Measures. Ph. D. Dissertation, Tilburg University, 2005.

[9] Bossert, W., Pattanaik, P.K., Xu, Y., Similarity of options and the measurement of diversity. Journal of Theoretical Politics 15 (4), pp. 405-421, 2003. 
[10] Cabrerizo, F.J., Moreno, J.M., Pérez, I.J., Herrera-Viedma, E., Analyzing consensus approaches in fuzzy group decision making: advantages and drawbacks. Soft Computing 14, pp. 451-463, 2010.

[11] Cabrerizo, F.J., Pérez, I.J., Herrera-Viedma, E., Managing the consensus in group decision making in an unbalanced fuzzy linguistic context with incomplete information. Knowledge-Based Systems 23, pp. 169-181, 2010.

[12] Coombs, C.H., A Theory of Data. Wiley, New York, 1964.

[13] Cox, T.F., Cox, M.A.A., Multidimensional Scaling, 2nd edition. Chapman and Hall, Boca Raton, 2001.

[14] Dietrich, F., List, C., Judgment aggregation by quota rules: majority voting generalized. Journal of Theoretical Politics 19 (4), pp. 391-424, 2007.

[15] Erdamar, B., García-Lapresta, J.L., Pérez-Román, D., Sanver, M.R., Measuring consensus in a preference-approval context. Information Fusion 17, pp. 14-21, 2014.

[16] Everitt, B.S., Landau, S., Leese, M., Cluster Analysis, 4th edition. Oxford University Press, New York, 2001.

[17] Fedrizzi, M., Kacprzyk, J., Nurmi, H., Consensus degrees under fuzzy majorities and fuzzy preferences using OWA (ordered weighted average) operators. Control and Cybernetics 22, pp. 71-80, 1993.

[18] García-Lapresta, J.L., Llamazares, B., Majority decisions based on difference of votes. Journal of Mathematical Economics 35, pp. 463-481, 2001.

[19] García-Lapresta, J.L., Pérez-Román, D., Measuring consensus in weak orders. In: E. Herrera-Viedma, J.L. García-Lapresta, J. Kacprzyk, H. Nurmi, M. Fedrizzi, S. Zadrózny (eds.), Consensual Processes, STUDFUZZ, vol. 267. Springer-Verlag, Berlin, 2011, pp. 213-234.

[20] García-Lapresta, J.L., Pérez-Román, D., Consensus-based hierarchical agglomerative clustering in the context of weak orders. Proceedings of the 2013 IFSA-NAFIPS Joint Congress, Edmonton, pp. 1010-1015, 2013.

[21] García-Lapresta, J.L., Pérez-Román, D., Consensus-based clustering under hesitant qualitative assessments. Fuzzy Sets and Systems, forthcoming.

[22] García-Lapresta, J.L., Pérez-Román, D., Falcó, E., Consensus reaching processes under hesitant linguistic assessments. Proceedings of the IS'2014 Conference. Warsaw, 2014.

[23] Grossi, D., Pigozzi, G., Judgment Aggregation: A Primer. Synthesis Lectures on Artificial Intelligence and Machine Learning. Morgan and Claypool, 2014. 
[24] Herrera, F., Herrera-Viedma, E, Martínez, L., A fuzzy linguistic methodology to deal with unbalanced linguistic term sets. IEEE Transactions on Fuzzy Systems 16 (2), pp. 354-370, 2008.

[25] Herrera, F., Herrera-Viedma, E., Verdegay, J.L., A model of consensus in group decision making under linguistic assessments. Fuzzy Sets and Systems 78, pp. 73-87, 1996.

[26] Herrera-Viedma, E., Cabrerizo, F.J., Kacprzyk, J., Pedrycz, W., A review of soft consensus models in a fuzzy environment. Information Fusion 17, pp. 4-13, 2014.

[27] Jain, A.K., Murty, M.N., Flynn, P.J., Data clustering: A review. ACM Computing Surveys 31 (3), pp. 264-323, 1999.

[28] Kacprzyk, J., Fedrizzi, M.: 'Soft' consensus measures for monitoring real consensus reaching processes under fuzzy preferences. Control and Cybernetics 15, pp. 309-323, 1986.

[29] Kacprzyk, J., Fedrizzi, M., A 'soft' measure of consensus in the setting of partial (fuzzy) preferences. European Journal of Operational Research 34, pp. 315-325, 1988.

[30] Kacprzyk, J., Fedrizzi, M., A 'human-consistent' degree of consensus based on fuzzy logic with linguistic quantifiers. Mathematical Social Sciences 18, pp. 275-290, 1989.

[31] Kacprzyk, J., Fedrizzi, M., Nurmi, H., Group decision making and consensus under fuzzy preferences and fuzzy majority. Fuzzy Sets and Systems 49, pp. 21-31, 1992.

[32] Krantz, D.H., Luce, R.D., Suppes, P., Tversky, A., Foundations of Measurement, vol. I. Academic Press, New York, 1971.

[33] Kruskal, J.B., Wish, M., Multidimensional Scaling. Sage, Newbury Park, 1978.

[34] List, C., The theory of judgment aggregation: an introductory review. Synthese 187 (1), pp. 179-207, 2012.

[35] Llamazares, B., The forgotten decision rules: Majority rules based on difference of votes. Mathematical Social Sciences 51, pp. 311-326, 2006.

[36] Llamazares, B., Pérez-Asurmendi, P., García-Lapresta, J.L., Collective transitivity in majorities based on difference in support. Fuzzy Sets and Systems 216, pp. 3-15, 2013.

[37] Martínez-Panero, M., Consensus perspectives: Glimpses into theoretical advances and applications. In: E. Herrera-Viedma, J.L. García-Lapresta, J. Kacprzyk, H. Nurmi, M. Fedrizzi, S. Zadrózny (eds.), Consensual Processes, STUDFUZZ, vol. 267, Springer-Verlag, Berlin, 2011, pp. 179-193. 
[38] Mongin. P., The doctrinal paradox, the discursive dilemma, and logical aggregation theory. Theory and Decision 73 (3), pp. 315-355, 2012.

[39] Palomares, I., Estrella, F.J., Martínez, L., Herrera, F., Consensus under a fuzzy context: taxonomy, analysis framework AFRYCA and experimental case of study. Information Fusion 20, pp 252-271, 2014.

[40] Roberts. F.S., Measurement Theory. Addison-Wesley Publishing Company, Reading, 1979.

[41] Shepard, R.N., The analysis of proximities: Multidimensional scaling with an unknown distance function. I. Psychometrika 27 (2), pp. 125-140, 1962.

[42] Spillman, B., Bezdek, J., Spillman, R., Development of an instrument for the dynamic measurement of consensus. Communication Monographs 46, pp. 1-12, 1979. 